\title{
Hydrogen peroxide enhanced ultrasound- fistulography in the assessment of enterocutaneous fistulas complicating Crohn's disease
}

G Maconi, F Parente, G Bianchi Porro

\begin{abstract}
BackgroundlAims-Proper management of enterocutaneous fistulas complicating Crohn's disease largely depends on the anatomical characteristics of the sinus tracks as well as the coexistence of complications such as abscesses and distal bowel stenosis. The aim of this prospective study was to evaluate the accuracy of a new technique (hydrogen peroxide enhanced ultrasound (US)-fistulography) compared with conventional $x$ ray fistulogram and/or surgical findings in the detection of Crohn's disease associated enterocutaneous fistulas.
\end{abstract}

Methods-Patients with known Crohn's disease and a suspicion of enterocutaneous fistulas were prospectively studied with this novel technique, conventional $x$ ray fistulogram, and barium radiography as well as with computed tomography whenever an abdominal abscess was suspected at US. In those undergoing surgery, intraoperative findings were also compared.

Results-Seventeen of 502 (3.4\%) consecutive patients with Crohn's disease seen over a ten month period had associated enterocutaneous fistulas and were enrolled. Hydrogen peroxide enhanced US-fistulography visualised the extent and configuration of fistula in all cases: 13 patients had a fistula arising from the ileum and two from the sigmoid colon, whereas in two there was no evidence of communication with intestinal loops; in contrast, conventional $\boldsymbol{x}$ ray fistulography missed a correct definition of the fistulous branches or communication with intestinal loops in $50 \%(4 / 8)$ and $36 \%(4 / 11)$ of patients respectively; barium radiography showed fistulas in two cases only. The presence of abscesses along or close to the sinus track, as well as the coexistence of intestinal stenosis, was correctly detected at US in all patients.

Conclusions-Hydrogen peroxide enhanced US-fistulography could be considered the diagnostic procedure of choice in Crohn's disease associated enterocutaneous fistulas, as it is at least as accurate, simple, and safe as conventional $x$ ray fistulogram, does not miss coexisting abdominal complications, and also provides information on the diseased bowel segments. In addition, it can be easily repeated over time in order to monitor the course of fistulas undergoing conservative treatment.

(Gut 1999;45:874-878)

Keywords: enterocutaneous fistulas; Crohn's disease; $x$ ray fistulogram; hydrogen peroxide enhanced US-fistulography

Enterocutaneous fistulas constitute one of the most troublesome intestinal complications of Crohn's disease, occurring in $8.7-21 \%$ of affected patients. ${ }^{1}$ Although their optimal management is still debated, ${ }^{2-9}$ there is no doubt that the accurate anatomical definition of fistulous tracks with their secondary and internal openings is essential for a correct therapeutical approach. Indeed, the presence of complex sinus tracks, the coexistence of distal bowel obstruction or abscesses close to or communicating with the fistulous tract all appear to be indicators of poor response to medical treatment. ${ }^{10}$

At present, the above information can only be obtained by conventional $x$ ray fistulogram, which, however, cannot be easily repeated and has been reported to be associated with dissemination of septic fistulous content ${ }^{11}$; moreover, it does not provide any information on the affected bowel segments, thus making it necessary to perform additional diagnostic examinations - for example, small bowel enteroclysis and/or barium enema-in order to plan the proper treatment.

Although conventional abdominal ultrasound (US) usually permits clear visualisation of the bowel segment involved as well as the detection of most intestinal complications of Crohn's disease such as stenosis, abscesses and enteroenteric fistulas, ${ }^{12-14}$ it has limited value in the visualisation of enterocutaneous sinus tracks. ${ }^{14}$ In recent years, the technical amelioration of sonographic devices along with the introduction of microbubble containing contrast agents, such as hydrogen peroxide, which enhance ultrasonographic signals, have greatly improved the imaging of intra-abdominal lesions. ${ }^{15}$ To date, however, apart from one preliminary report suggesting the potential usefulness of the injection of sonographic contrast medium during rectal US for defining perianal fistulas, ${ }^{16}$ no controlled study has compared the accuracy of abdominal US with

Abbreviation used in this paper: US, ultrasound.
Accepted for publication 12 June 1999 
Table 1 Clinical and biochemical characteristics of the enrolled patients

\begin{tabular}{|c|c|c|}
\hline Variable & Number & $\begin{array}{l}\text { Median } \\
\text { (range) }\end{array}$ \\
\hline $\operatorname{Sex}(M / F)$ & $10 / 7$ & \\
\hline Age $(y)$ & & $38(23-46)$ \\
\hline Disease duration (months) & & $125(9-191)$ \\
\hline \multicolumn{3}{|l|}{ Disease location } \\
\hline Ileum and colon & 13 & \\
\hline Ileum only & 4 & \\
\hline Disease activity (CDAI) & & $191(48-300)$ \\
\hline C-reactive protein (mg/l) & & $40(7-200)$ \\
\hline ESR (mm, first hour) & & $30.5(10-92)$ \\
\hline \multicolumn{3}{|l|}{ Location of external orifice } \\
\hline Right iliaca fossa & 7 & \\
\hline Median laparotomy scar & 9 & \\
\hline Peristomal & 1 & \\
\hline \multicolumn{3}{|l|}{ Nature of the fistulas } \\
\hline Post-surgical & 16 & \\
\hline Spontaneous & 1 & \\
\hline $\begin{array}{l}\text { Time elapsed since surgery } \\
\text { (months) }\end{array}$ & & $3(1-15)$ \\
\hline
\end{tabular}

that of conventional techniques for the assessment of enterocutaneous fistulas associated with Crohn's disease.

We have therefore undertaken a prospective study with two specific aims: (a) to assess the accuracy of hydrogen peroxide enhanced US-fistulography in the visualisation of fistulous tracks as compared with conventional $x$ ray fistulogram and/or with surgical findings in a consecutive series of patients with enterocutaneous fistulas complicating Crohn's disease; (b) to evaluate the usefulness of this technique in monitoring the course of fistulas in patients undergoing conservative treatment.

\section{Materials and methods}

PATIENTS

Consecutive patients with known Crohn's disease of the large and/or small bowel with a suspicion of enterocutaneous fistulas who either attended the gastroenterological outpatient clinic or were admitted as inpatients to the Department of Gastroenterology of L Sacco University Hospital over a ten month period (September 1997 to April 1998) were invited to participate in this prospective study. In all patients, Crohn's disease had been verified by standard criteria ${ }^{17}$ and the anatomic extension of the disease was determined on the basis of total colonoscopy (or double contrast barium enema if colonoscopy was not successful), small bowel enteroclysis, and conventional abdominal US performed at the time of study enrolment. The criteria for admission to the study also required that all patients were more than 18 years old and had accurate medical records of clinical, anatomical, and therapeutical characteristics of his/her disease. In particular, details were sought about the duration of disease, extraintestinal complications, current and previous medical treatment, as well as previous surgery; in addition, disease activity was determined at the time of study admission in accordance with a scale consisting of the Crohn's disease activity index. ${ }^{18}$

All patients admitted underwent USfistulography (see below) and conventional $x$ ray fistulogram within a maximum interval of one week. Examiners were informed about the clinical findings but were blinded to the results of the other investigation. In those patients in whom conventional $x$ ray fistulogram was not feasible, the results of US-fistulography were compared with the surgical findings only.

\section{PROCEDURE}

All patients underwent conventional US examination which was carried out by the same two doctors (GM and FP). Sonographic scans of the whole abdomen were carried out with a real time ultrasonic apparatus (Ecotron-Aloka SSD 680). The examination was begun with a convex $3.5 \mathrm{MHz}$ transducer and, for a detailed evaluation, a linear $7.5 \mathrm{MHz}$ transducer was then used. No special preparation, such as fasting, administration of spasmolytic agents, or water instillation into the bowel, was performed. An intestinal wall thickness of $4 \mathrm{~mm}$ or more was considered pathological and was evaluated with respect to maximum wall thickness observed, extent, and location. The maximum bowel wall thickness was measured in both longitudinal and transverse sections, and only those measurements that could be reproduced for at least $4 \mathrm{~cm}$ in length were considered. ${ }^{12} 14$

Stenosis was considered present at US when there was the coexistence of thickened and stiff bowel wall, narrowed intestinal lumen along with distended fluid, or echogenic content-

Table 2 Description of fistulas at ultrasound (US), US-fistulography (US-F), x ray fistulogram (Xry-F) and surgery

\begin{tabular}{|c|c|c|c|c|c|c|c|c|c|c|c|c|c|c|}
\hline \multirow[b]{2}{*}{ Patient no } & \multirow[b]{2}{*}{ Sex } & \multirow[b]{2}{*}{ Age (y) } & \multicolumn{4}{|c|}{ Patency } & \multicolumn{4}{|c|}{ Internal opening } & \multicolumn{4}{|c|}{ Branches } \\
\hline & & & $U S$ & $U S-F$ & $X r y-F$ & Surgery & $U S$ & $U S-F$ & $X r y-F$ & Surgery & $U S$ & $U S-F$ & $X r y-F$ & Surgery \\
\hline 1 & M & 45 & Yes & Yes & & Yes & Sigma & Sigma & & Sigma & No & No & & No \\
\hline 2 & $\mathrm{M}$ & 43 & Yes & Yes & & Yes & Ileum & Ileum & & Ileum & No & No & & No \\
\hline 3 & $\mathrm{~F}$ & 46 & Yes & Yes & & Yes & Ileum & Ileum & & Caecum & No & No & & No \\
\hline 4 & $\mathrm{~F}$ & 38 & No & Yes & & Yes & Ileum & Ileum & & Ileum & Yes & Yes & & Yes \\
\hline 5 & $\mathrm{M}$ & 37 & Yes & Yes & Yes & Yes & Ileum & Ileum & Ileum & Ileum & Yes & Yes & No & Yes \\
\hline 6 & M & 40 & Yes & Yes & Yes & Yes & Caecum & Ileum & Ascending colon & Ileum & Yes & Yes & Yes & Yes \\
\hline 7 & $\mathrm{M}$ & 24 & Yes & Yes & Yes & Yes & Caecum & Ileum & No & Caecum & Yes & Yes & Yes & Yes \\
\hline 8 & $\mathrm{M}$ & 37 & No & Yes & Yes & & No & Ileum & Ileum & & No & No & No & \\
\hline 9 & $\mathrm{M}$ & 32 & No & Yes & Yes & & Ileum & Ileum & No & & No & No & No & \\
\hline 10 & $\mathrm{M}$ & 34 & No & Yes & Yes & & Ileum & Ileum & Ileum & & No & No & No & \\
\hline 11 & $\mathrm{~F}$ & 31 & No & Yes & Yes & & Sigma & Sigma & Sigma & & Yes & Yes & Yes & \\
\hline 12 & $\mathrm{~F}$ & 28 & No & Yes & Yes & & Ileum & Ileum & No & & No & No & No & \\
\hline 13 & $\mathrm{~F}$ & 36 & No & Yes & Yes & & No & Ileum & No & & No & Yes & Yes & \\
\hline 14 & $\mathrm{~F}$ & 27 & No & Yes & Yes & & No & Ileum & Ileum & & No & No & No & \\
\hline 15 & $\mathrm{M}$ & 46 & No & Yes & Yes & & No & Ileum & Ileum & & No & No & No & \\
\hline 16 & M & 23 & No & Yes & Yes & & No & No & No & & No & Yes & No & \\
\hline 17 & $\mathrm{~F}$ & 41 & No & Yes & Yes & & No & No & No & & Yes & Yes & No & \\
\hline
\end{tabular}


filled loops just above the thickened intestinal segment, as previously described. ${ }^{13}$

A suspicion of enterocutaneous fistula was defined as the presence of a hypoechoic or anechoic duct-like structure with or without fluid and/or air content, localised between the skin and the intestinal loops, whereas concomitant abscesses were indicated by the presence of anechoic or hypoechoic round masses, of at least $2 \mathrm{~cm}$ in diameter, with or without air bubbles inside, close to, or communicating with the fistulous branches. Computed tomography of the whole abdomen was performed in all patients in whom abscesses were suspected at conventional US one hour after oral administration of $500 \mathrm{ml} 0.5 \%$ iopaminole solution and with simultaneous intravenous bolus injection of $100 \mathrm{ml}$ non-ionising contrast medium.

To provide adequate visualisation of the fistulous track and to ascertain its communication with an intestinal loop or other abdominal cavities, $20-90 \mathrm{ml}$ of a solution (according to the output of the fistula), consisting of $30 \%$ hydrogen peroxide and $70 \%$ povidone/iodine, was slowly injected via a flexible venflon into the skin orifice of the fistula. This concentration of hydroxide peroxide had already been shown to produce good US visualisation of fistulous tracks in a previous series of patients (unpublished data). Ultrasound images were videotaped before, during, and after the injection of sonographic contrast medium and photographically documented.

Conventional $x$ ray fistulogram was performed within a maximum interval of one week from US-fistulography by inserting a fine catheter into the skin opening of the fistula and injecting contrast medium. Films were taken in two planes to attempt to localise the site of the fistula.

The accuracy of hydrogen peroxide USenhanced fistulography in delineating enterocutaneous fistulas was compared with that of conventional US, $x$ ray fistulogram, and, in patients undergoing surgical treatment, also with intraoperative findings.

In particular, the results of this procedure were evaluated according to the following relevant information: (a) accurate determina-

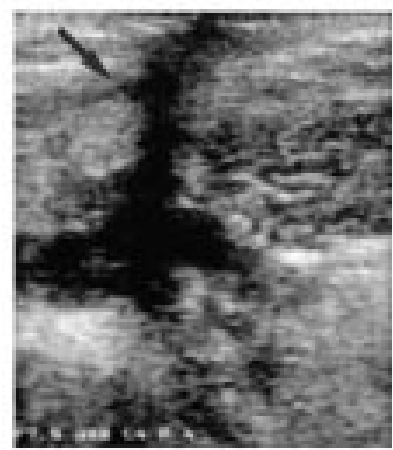

A

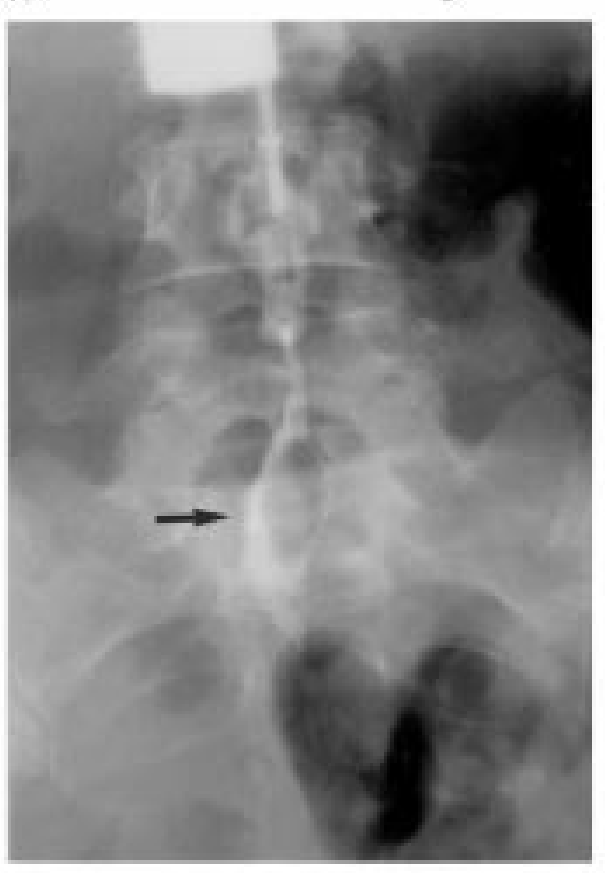

D

B
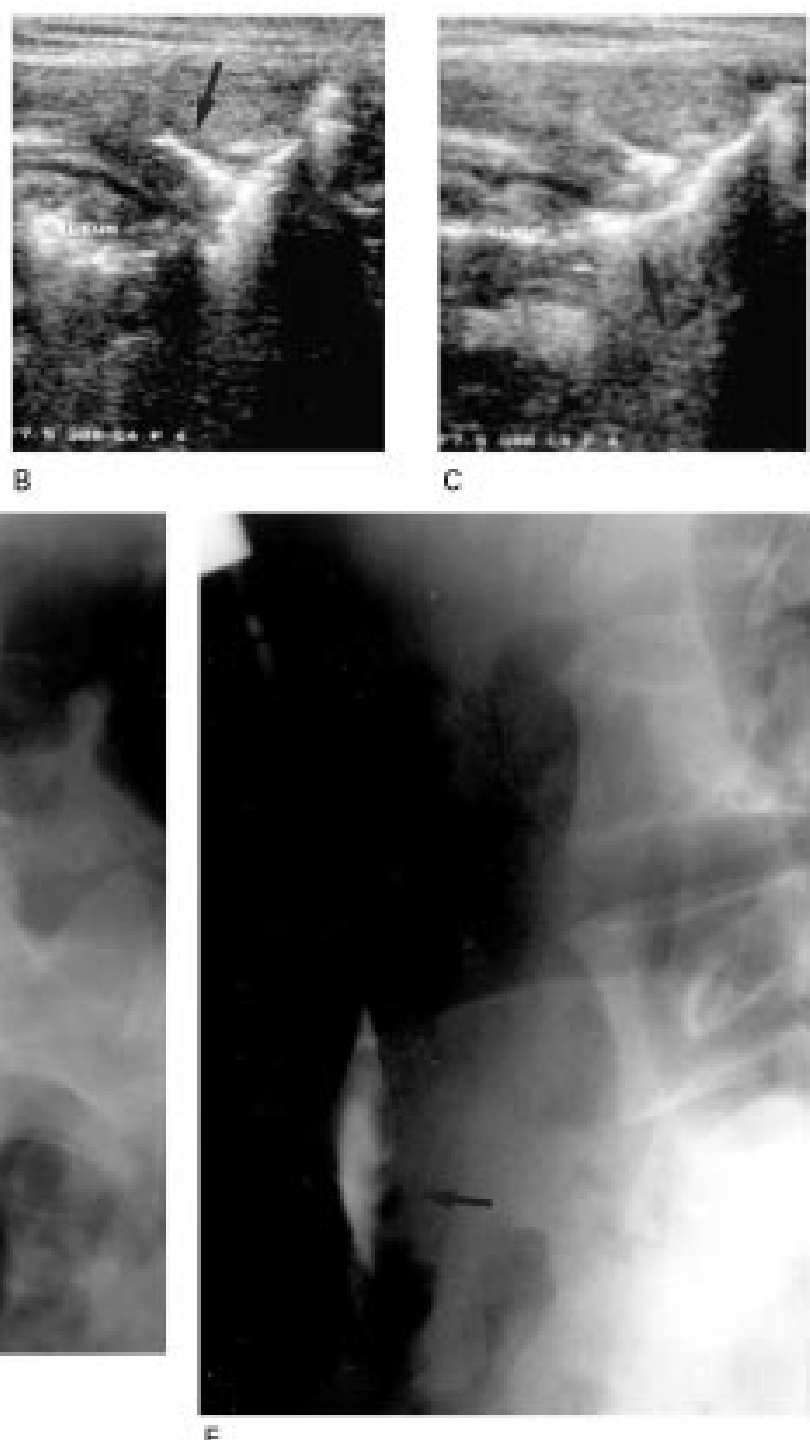

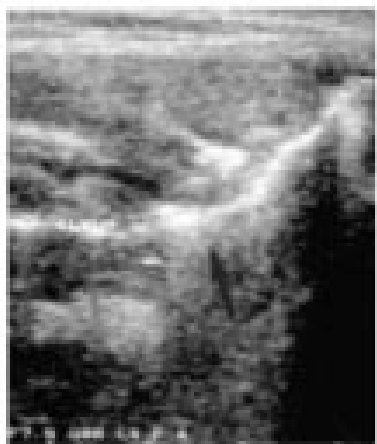

c

Figure 1 Enterocutaneous fistula which appears to be simple (arrow) at conventional US (A). Injection of the hydrogen peroxide solution into the sinus track discloses a complex sinus track $(B, C)$, with two major branches (arrow) and a clear communication with an ileal loop (arrow) (C). Conventional x ray fistulogram shows only a simple sinus track (arrow) apparently not in communication with the bowel $(D, E)$. 
Table 3 Associated fistula complications (intestinal strictures, internal fistulas, abscesses) as detected by ultrasound (US), barium radiography ( $x$ ray studies) and computed tomography $(C T)$

\begin{tabular}{|c|c|c|c|c|c|c|c|c|}
\hline \multirow[b]{2}{*}{$\begin{array}{l}\text { Patient } \\
\text { no }\end{array}$} & \multirow[b]{2}{*}{ Sex } & \multirow[b]{2}{*}{$\begin{array}{c}\text { Age } \\
(y)\end{array}$} & \multicolumn{2}{|c|}{ Strictures } & \multicolumn{2}{|c|}{ Internal fistulas } & \multicolumn{2}{|c|}{ Abscesses } \\
\hline & & & US & $\begin{array}{l}x \text { ray } \\
\text { studies }\end{array}$ & $U S$ & $\begin{array}{l}x \text { ray } \\
\text { studies }\end{array}$ & $U S$ & $C T$ \\
\hline 1 & $M$ & 45 & Yes & Yes & Yes & Yes & No & No \\
\hline 2 & $M$ & 43 & Yes & Yes & Yes & Yes & Yes & Yes \\
\hline 3 & $\mathrm{~F}$ & 46 & Yes & Yes & No & No & No & NA \\
\hline 4 & $\mathrm{~F}$ & 38 & Yes & Yes & Yes & Yes & Yes & Yes \\
\hline 5 & $M$ & 37 & No & No & No & No & Yes & Yes \\
\hline 6 & $M$ & 40 & No & No & No & Yes & No & NA \\
\hline 7 & $M$ & 24 & Yes & Yes & Yes & Yes & No & NA \\
\hline 8 & $M$ & 37 & No & No & No & No & No & NA \\
\hline 9 & $M$ & 32 & No & No & No & No & No & NA \\
\hline 10 & $M$ & 34 & Yes & Yes & No & No & No & NA \\
\hline 11 & $\mathrm{~F}$ & 31 & Yes & Yes & Yes & Yes & No & NA \\
\hline 12 & $\mathrm{~F}$ & 28 & No & No & No & No & No & NA \\
\hline 13 & $\mathrm{~F}$ & 36 & No & No & No & Yes & No & No \\
\hline 14 & $M$ & 23 & No & No & No & No & No & NA \\
\hline 15 & $M$ & 46 & No & No & No & No & Yes & Yes \\
\hline 16 & $\mathrm{~F}$ & 27 & No & No & No & No & Yes & Yes \\
\hline 17 & $\mathrm{~F}$ & 41 & No & No & No & No & No & NA \\
\hline
\end{tabular}

NA, not available.

tion of the fistula anatomy, with its primary and secondary openings; (b) connection to an intestinal loop; (c) connection to other abdominal organs; (d) coexistence of abscesses close to or communicating with the sinus tracks.

In patients undergoing conservative treatment, conventional US and hydrogen peroxide US-enhanced fistulography were repeated by the same operators at monthly intervals until the fistula was healed or for a maximum follow up of six months.

The study was approved by the L Sacco Hospital ethics committee, and informed consent was obtained from all patients undergoing US-fistulography.

\section{Results}

Enterocutaneous fistulas were diagnosed in 17 $(3.4 \%)$ of 502 consecutive patients with known Crohn's disease of the large and/or small bowel, seen at out institution from September 1997 to April 1998. In five patients, the fistula was already diagnosed clinically and it was reconfirmed during the study period. In six patients with a previous diagnosis of enterocutaneous fistulas, no cutaneous opening of the fistula was present at the time of investigation and therefore the diagnosis was not established.

Table 1 gives the demographic and clinical characteristics of the enrolled patients. All these patients were examined by conventional abdominal US and agreed to undergo hydrogen peroxide enhanced US-fistulography. The results of the latter procedure were verified by conventional $x$ ray fistulogram in 13 patients and by surgery in seven. In particular, in four patients, $x$ ray fistulogram was not successful because of notable inflammatory infiltration of the periorificial skin and/or the development of severe pain at injection of the radio-opaque medium contrast into the sinus track.

Table 2 summarises the results of USfistulography as compared with $x$ ray fistulogram and surgical findings in visualising the fistulous tracks. Conventional US showed the presence of a sinus track between the skin and intestinal loops in all patients with diagnosed enterocutaneous fistulas and the presence of abscesses along or close to the fistula branches in four cases. However, only in five cases was the passage of fluid echogenic material throughout the sinus track documented by this technique, thus confirming with certainty the patency of the fistula. In contrast, hydrogen peroxide US-fistulography showed the exact extension and configuration of the fistulas, with their primary and secondary openings, in all cases (fig 1). In particular, a clear communication between the sinus track and intestinal loops was clearly visible during or immediately after the injection of sonographic medium contrast in 15 patients, whereas in two cases there was no evidence of communication with the bowel. In contrast, $x$ ray fistulogram showed fistulas opening into an intestinal loop in seven out of 11 patients $(64 \%)$.

Five patients in whom an abdominal abscess was suspected at conventional US underwent a computed tomography scan of the whole abdomen, and subsequently four of them underwent surgery. Table 3 gives the accuracy of US findings as compared with computed tomography, small bowel enteroclysis, colonoscopy or barium enema in the detection of concomitant intestinal complications such as abscesses and stenosis, as well as other information on the affected bowel segments.

Eight patients with enterocutaneous fistulas and moderate to severe Crohn's disease activity but without intestinal complications were treated with immunosuppressive agents (five with azathioprine $2 \mathrm{mg} / \mathrm{kg}$ per day and three with 6-mercaptopurine $25 \mathrm{mg}$ intravenously once a week). All these patients were regularly followed up, at monthly intervals, by conventional US and hydrogen peroxide USfistulography until the closure of the fistula or for a maximum period of six months. In two patients, closure of enterocutaneous fistulas occurred during the follow up, whereas in another the fistula initially closed and subsequently reopened with the development of a small abscess along the sinus track. The behaviour of these fistulas during the follow up was well documented by US-fistulography.

No serious complications occurred during the procedure or afterwards. Only moderate hyperalgesia at the injection site and mild and transient abdominal pain occurred during the injection of the hydrogen peroxide solution in three and two patients respectively.

\section{Discussion}

The proper management of enterocutaneous fistulas complicating Crohn's disease is still under debate, as different treatments, including early surgical resection, ${ }^{2}$ bowel rest with parenteral hyperalimentation, ${ }^{34}$ control of local sepsis, ${ }^{5}$ immunosuppressive treatment ${ }^{6-8}$ and, more recently, the use of antibody preparations directed towards tumour necrosis factor ${ }^{9}$ have been advocated by various authors. However, it is well established that one of the mainstays of successful treatment of this condition is the early definition of the site, the anatomy and nature of the fistula, and the identification of any factor likely to prevent 
spontaneous closure. Indeed, spontaneous and complex sinus tracks, especially when complicated by abscesses close to or communicating with the fistulous tract or by distal bowel stenosis, are less prone to undergo spontaneous closure than postoperative and simple fistulas without clinical evidence of intra-abdominal sepsis. ${ }^{5}$

Until now, enterocutaneous fistulas have been detected, for the most part, by conventional $x$ ray fistulogram or, incidentally, by barium follow through or double contrast barium enema, thanks to retrograde filling of the sinus tract; the former technique, however, requires $x$ ray exposure, may be associated with dissemination of septic fistulous content and, moreover, does not provide information about those associated complications that are predictors of poor response to medical treatment nor about the affected bowel loops, which is necessary for planning proper treatment.

Our paper shows the value of a new technique based on the injection of a sonographic contrast medium into the sinus tract to obtain ultrasonic definition of the fistula anatomy. This technique has proved to be more accurate than $x$ ray fistulogram because of detailed visualisation of the extent of the lesions, the ability to detect all the sinus branches and internal openings, and the accurate topographic mapping of Crohn's disease. Indeed, conventional $x$ ray fistulogram in comparison with US-fistulography failed to show the correct definition of the fistulous branches in four of eight $(50 \%)$ patients and failed to show communications with intestinal loops in four of eleven $(36 \%)$ cases. The presence of abscesses along or close to the sinus track, as well as the coexistence of intestinal stenosis, was also correctly detected at US in all patients.

The high accuracy of US-fistulography is also supported by surgical findings and pathology specimens in patients undergoing surgery, where there was good correspondence to the fistula configuration as determined by US.

One of the major advantages of this new technique is that it requires little effort, as it may be performed during conventional US examination of the abdomen; it is also well tolerated by the patients and avoids radiation exposure so it can be easily repeated. In addition, there is no need for any special preparation for the examination or the use of radio-opaque medium and no risk of septic dissemination of fistulous content, as the sinus tracks are irrigated with disinfectants. Another merit of this technique is that it not only permits the diagnosis of fistulas in cases that are not clear clinically, but it also provides detailed information on the affected bowel segments which otherwise could only be obtained by performing barium radiography or colonoscopy. Indeed, transabdominal US gave results comparable with small bowel enteroclysis or barium enema/colonoscopy in evaluating the anatomical distribution of Crohn's disease, the length of bowel involved, and the presence of stenosis, in agreement with our previous results. ${ }^{12}$

Conservative management of enterocutaneous fistulas can also be improved by this technique, based on repeated objective imaging of the lesions. This is of particular importance if we consider the potential efficacy of new therapeutical modalities-for example, the use of anti-tumour necrosis factor monoclonal antibodies-which will probably expand the indications for medical treatment of this condition in the near future. ${ }^{9}$

In conclusion, hydrogen peroxide enhanced US-fistulography is a very accurate, simple, and safe diagnostic method of visualising enterocutaneous fistulas associated with Crohn's disease. This single technique provides information comparable with that obtained previously only by combining the results of multiple procedures, such as conventional $x$ ray fistulogram and barium radiography. For these reasons we believe that it will become the standard diagnostic imaging technique for staging enterocutaneous fistulas.

1 Korelitz B, Felder J. Gastrointestinal complications of ulcerative colitis and Crohn's disease. In: Kirsner JB, ulcerative colitis and Crohn's disease. In: Kirsner JB, Shorter RG, eds. Inflammatory bowel disease.
more: Williams \& Wilkins, 1995:437-60.

2 Hawker P, Givel J, Keighley M, et al. Management of enterocutaneous fistulae in Crohn's disease. Gut 1983;24:2847.

3 Muller J, Hargrave W, Dudrick S, et al. Ten years experience with intravenous hyperalimentation and inflammatory bowel disease. Ann Surg 1978;187:523-9.

4 Driscoll R Jr, Rosenberg I. Total parenteral nutrition in inflammatory bowel disease. Med Clin N Am 1978;6:185201.

5 McIntyre PB, Ritchie JK, Hawley PR, et al. Management of enterocutaneous fistulas: a review of 132 cases. Br $\mathcal{F}$ Surg 1984;71:293-6.

6 Korelitz BI, Present DH. Favourable effect of 6-mercaptopurine on the fistulae of Crohn's disease. Dig Dis Sci 1985;30:58-64.

7 Korelitz B, Adler D, Mendelson R, et al. Long-term experience with 6-mercaptopurine in the treatment of Crohn's disease. Am f Gastroenterol 1993;88:1198-1205.

8 Sandborne WJ. A critical review of cyclosporine therapy in inflammatory bowel disease. Inflammatory Bowel Disease 1995;1:48-63.

9 Present D, Mayer L, VanDeventer SJ, et al. Anti-TNF-alpha chimeric antibody (cA2) is effective in the treatment of the fistulae of Crohn's disease: a multicenter, randomised, double-blind, placebo-controlled study. Am f Gastroenterol 1907; $92: 1746$.

10 Greenberg N, Miner P Jr. Indications for surgery in inflammatory bowel disease: a gastroenterologist's opinion. In: Kirsner JB, Shorter RG, eds. Inflammatory bowel disease. 4th ed. Baltimore: Williams \& Wilkins, 1995:750-70.

11 Tio TL, Mulder JJ, Wijers OB, et al. Endosonography of peri-anal and peri-colorectal fistula and/or abscess in Crohn's disease. Gastrointest Endosc 1990;36:331-6.

12 Maconi G, Parente F, Bollani S, et al. Abdominal ultrasound in the assessment of extent and activity of Crohn's disease: clinical significance and implication of bowel wall thickening. Am f Gastroenterol 1996;91:1604-9.

13 Maconi G, Bollani S, Bianchi Porro G. Ultrasonographic detection of intestinal complications in Crohn's disease. Dig Dis Sci 1996;41:1643-8.

14 Gasche C, Moser G, Turetsechek K, et al. Prospective evaluation of transabdominal bowel sonography for detection of specific intestinal complications in Crohn's disease [abstract]. Gastroenterology 1997;112:A980.

15 Goldberg B, Liu J. Contrast agents in abdominal ultrasound. Frontiers of Gastrointestinal Research 1997;24:32341.

16 Poen AC, Felt-Bersma RJ, Eijsbouts QA, et al. Hydrogen peroxide assisted transanal ultrasound in the diagnosis of fistula-in ano [abstract]. Gut 1997;41(suppl 3):A17.

17 Malchow H, Ewe K, Brandes JW, et al. European Malchow H, Ewe K, Brandes JW, et al. European
cooperative Crohn's disease study (ECCDS): results of cooperative Crohn's disease study (ECCDS): r
drug treatment. Gastroenterology 1984;86:249-66.

18 Best W, Becketel J, Singleton J, et al. Development of a Crohn's disease activity index; national cooperative Crohn's disease study. Gastroenterology 1976;70:439-44. 